\title{
Improving the quality of handover: implementing SBAR
}

\author{
Authors: Zeinab Ruhomauly, ${ }^{\mathrm{A}}$ Kathryn Betts, ${ }^{\mathrm{A}}$ Katherine Jayne-Coupe, ${ }^{\mathrm{A}}$ Luciné Karanfilian, ${ }^{\mathrm{A}}$ Megan Szekely, ${ }^{\mathrm{A}}$ \\ Anu Relwani, ${ }^{B}$ Joel McCay ${ }^{B}$ and Zahra Jaffry ${ }^{B}$
}

\section{Introduction}

Effective communication is essential in the provision of safe patient care. Use of structured communication tools, such as the Situation, Background, Assessment and Recommendation (SBAR) format of handover have been shown to improve patient safety, especially for telephonic handovers. ${ }^{1}$ SBAR has widely been recommended as a standardised method of handover. ${ }^{2,3}$ We undertook a quality improvement (QI) project to increase SBAR awareness across two wards in a district general hospital. This study describes the effect of our interventions and the challenges of implementing QI methodology to measuring safe communication and handover.

\section{Materials and methods}

A baseline audit was conducted to assess awareness and understanding of SBAR. Additionally, structured interviews were conducted with senior nurses and simulation training officers to understand barriers to SBAR use. One nurse on each ward was appointed as an 'SBAR champion'. We implemented 10-minute ward-based teaching sessions, which the champions then continued. Posters and telephone cards were implemented to reinforce teaching.

Outcomes measured included:

$>\%$ of nurses reporting exclusively using SBAR as their method of handover

$>\%$ of nurses aware of SBAR

$>$ perceived effectiveness of giving telephonic handovers (selfreported).

\section{Results and discussion}

Implementation of SBAR champions, teaching sessions and visual aids on the wards demonstrated the following results:

$>54.4 \%$ improvement in the proportion of nurses reporting using exclusively SBAR as their method of handover

$>100 \%$ of nursing staff were aware of SBAR (improved from a baseline of $87.5 \%$ )

$>44 \%$ average improvement in the self-reported perceived effectiveness of telephone handovers.

Although the results demonstrate a clear improvement in awareness and understanding of SBAR, the use of subjective data presents limitations. Objectively assessing the quality and effectiveness of SBAR handovers requires controlled, simulated environments, which are difficult methods to replicate in real-world settings using QI methodology. ${ }^{4}$ Despite this, our interventions demonstrate an improvement in SBAR awareness and can easily be replicated across other wards. Additionally, our interventions were well-researched and were developed following the identification of key barriers to SBAR use, from questionnaires and through structured interviews. Engaging senior nursing staff across the trust proved successful in appointing SBAR champions across the wards, ensuring that the teaching intervention remains sustainable.

\section{Conclusion}

Ward-based teaching sessions and visual aids may offer effective and scalable methods of increasing awareness and understanding of the SBAR communication tool for handovers. Ultimately, strengthening communication requires engaging senior staff members to promote good handover culture. Our methods demonstrate the difficulties in objectively measuring handover outcomes and adverse patient outcomes. Although good communication represents a crucial component of patient safety and ensuring good quality care, therefore forming an important aspect of QI, lack of objective measures may present difficulties in applying sound QI methodology.

\section{References}

1 Müller $M$ et al. Impact of the communication and patient handoff tool SBAR on patient safety: a systematic review. BMJ Open 2018:8:e022202.

2 Royal College of Physicians. Improving teams in healthcare - Team communication. London: RCP, 2017.

3 World Health Organization. Communication during patient handovers. WHO, 2007.

4 Cunningham N], Weiland T], van Dijk ] et al. Telephone referrals by junior doctors: a randomised controlled trial assessing the impact of SBAR in a simulated setting. Postgraduate Medical Journal 2012;88:619-26.

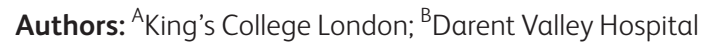

